\title{
Las virtudes de la humanidad, justicia y moderación y su relación con la conducta sustentable
}

\section{Virtues of humanity, justice and temperance and their relationship with sustainable behavior}

Recibido: 08/07/2013

Revisado: $12 / 08 / 2013$

Aceptado: 25/10/2013

\begin{abstract}
Based upon the idea that sustainable (prosocial, proenvironmental) actions are a kind of virtuous behavior, this study explores the relationship between three universal virtues -Humanity, Justice, Temperance - and four instances of sustainable behavior (SB) -altruistic, proecological, frugal and equitable actions - which are considered necessary for achieving the ideals of sustainable development. The responses of four hundred individuals, living in a northwestern Mexican city to the Inventory of Strengths and Virtues (VIA Survey, Peterson \& Seligman, 2004), as well as an instrument assessing sustainable behavior, were processed within a structural equation model. This model produced first-order factors for the three analyzed virtues and the four types of sustainable behaviors. In turn, the group of virtues generated a second-order factor (Virtue) and the group of sustainable behaviors produced a secondorder factor (SB) as well. The covariation between the Virtue
\end{abstract}

\author{
Víctor Corral-Verdugo, \\ César Tapia-Fonllem, \\ Anais Ortiz-Valdez y \\ Blanca Fraijo-Sing \\ Universidad de Sonora, México
}

Reconocimientos: Proyecto financiado por el Consejo Nacional de Ciencia y Tecnología (Conacyt 179886).

Agradecimientos: Los autores agradecen a Martin Seligman y Neal Mayerson su autorización para utilizar el instrumento VIA de fortalezas y virtudes. También a Kelly Aluise, quien gentilmente nos auxilió en el proceso de codificación y calificación de ese instrumento.

Correspondencia: Blvd. Luis Encinas y Rosales S/N, Hermosillo, Sonora, 83000, México. E-mail: victorcorral@sociales.uson.mx.

\section{Resumen}

Basado en la idea de que la conducta sustentable es un tipo de comportamiento virtuoso, el presente estudio explora la relación entre tres virtudes universales - Humanidad, Justicia, Moderación - y cuatro instancias de conducta sustentable - acciones altruistas, pro-ecológicas, frugales y equitativas - las cuales se consideran necesarias para alcanzar los ideales de un desarrollo sostenible. Se investigaron las respuestas que dieron cuatrocientas personas de la población general, en una ciudad del noroeste mexicano, a reactivos del Inventario de Virtudes y Fortalezas Psicológicas (VIA Survey, Peterson \& Seligman, 2004) y a un instrumento que investigaba conductas sustentables. Los resultados se procesaron dentro de un modelo de ecuaciones estructurales, el cual produjo factores de primer orden para las tres virtudes y los cuatro tipos de comportamiento sustentables. A su vez, el grupo de virtudes generó un factor de segundo orden (virtudes) y eso mismo ocurrió para el de conductas 
and SB factors was salient and significant, which seems to indicate that prosocial and proenvironmental behaviors have a virtuous nature, at least in regard to Humanity, Justice, and Temperance.

Key words: humanity, justice, temperance, sustainable behavior

La conducta sustentable (CS) -el conjunto de acciones encaminadas a la protección de los recursos naturales y socioculturales del planeta- goza de un estatus de amplia aceptación y su promoción es visible en la mayoría de las sociedades actuales. De acuerdo con Corral (2012) esta característica hace aparecer a la CS como un tipo de conducta virtuosa. La CS incluye comportamientos de cuidado del medio ambiente físico, tales como las acciones pro-ecológicas y aquellas que combaten el consumismo y la depredación de recursos naturales; pero además incluye comportamientos que buscan atender, empoderar y satisfacer las necesidades de otros, como se da en el caso de los actos altruistas y equitativos (Tapia, Corral, Fraijo \& Durón, 2013). La conexión teórica entre conducta sustentable y las virtudes se da, en buena medida, porque la CS cumple con prácticamente todas las características que identifican a las acciones virtuosas (Corral, 2012).

Las virtudes son manifestaciones de "excelencia o fortalezas de carácter que hacen posible a los individuos florecer como seres humanos" (Fowers, 2005, p. 4). Para Peterson y Seligman (2004), las virtudes son blancos deliberados de las prácticas sociales que procuran cultivarlas; proporcionan plenitud y satisfacción individual, son valoradas por derecho propio, se encuentran notoriamente encarnadas en algunos individuos, elevan a quienes las atestiguan produciendo admiración, y son ampliamente reconocidas en todas las culturas.

Es posible especular acerca de la naturaleza virtuosa de la CS dada una serie de evidencias reportadas en la literatura. El comportamiento sustentable es promovido y cultivado (a partir de la educación ambiental y para la sustentabilidad) por sociedades y gobierno en todo el mundo (Dobson, 2007; Roseland, 2012); de aquí se desprende que la CS sea también valorada por derecho propio, como lo establecen Peterson y Seligman (2004). La conducta sustentables (CS). La covariación entre el factor superior de virtudes y el de CS fue alta y significativa lo que parece indicar que las conductas prosociales y proambientales son de naturaleza virtuosa, por lo menos en lo concerniente a las tres virtudes aquí analizadas.

Palabras clave: humanidad, justicia, moderación, conducta sustentable

sustentable, al igual que las virtudes, genera satisfacción individual y otros tipos de beneficios psicológicos como la motivación intrínseca, felicidad y bienestar personal (Brown \& Kasser, 2005; De Young, 1996). Adicionalmente, y al igual que las virtudes, la conducta sustentable se presenta más en ciertos individuos que en otros (Tapia et al., 2013) $\mathrm{y}$ es ampliamente reconocida en prácticamente todas las culturas (Corral, 2012).

Las virtudes comparten con algunos valores (objetivos deseables que sirven como principios que guían la vida de las personas) su potencial influencia en la conducta sustentable (Steg \& de Groot, 2012). De hecho, algunos valores humanos juegan un rol importante en las acciones proambientales (Dunlap, Grieneeks \& Rokeach, 1983). Por ejemplo, los valores altruistas y, sobre todo, los biosféricos, predicen creencias, normas y conductas proambientales (De Groot \& Steg, 2007; Nilsson, von Borgstede \& Biel, 2004); alternativamente, los valores hedónicos se relacionan negativamente con la conducta sustentable (Steg, Vlek \& Slotegraaf, 2001).

No obstante las coincidencias planteadas entre la acción sustentable y las virtudes, no existe investigación sistemática que determine de manera empírica las relaciones entre virtudes y conducta sustentable. Consecuentemente, el propósito del presente artículo es ofrecer los resultados de un estudio en el que se prueba la hipótesis de una alta covariación entre los dos tipos de constructos. De manera particular, fue interés del estudio que aquí se reporta explorar las posibles asociaciones entre la conducta sustentable y tres tipos de virtudes: la Humanidad, la Justicia y la Moderación, dadas sus aparentes similitudes con los objetivos que pretende alcanzar la CS. Previamente se efectúa una breve revisión de la literatura que parece apoyar la idea de una significativa asociación entre estas tres virtudes y las conductas sustentables. 


\section{Cuatro instancias de la conducta sustentable}

Corral, Frías y García (2010) proponen que la conducta sustentable se compone por lo menos de cuatro categorías comportamentales. Dos de ellas, la conducta pro-ecológica y el comportamiento frugal se enfocan más hacia la conservación de los recursos naturales, mientras que las otras dos, la conducta altruista y las acciones equitativas, se relacionan preferencialmente con el cuidado dirigido hacia otros seres humanos. Aun así, se presentan interrelaciones sobresalientes y significativas entre los cuatro tipos de comportamiento, lo que permite suponer la existencia de un factor de segundo orden, al que los investigadores identifican como "conducta sustentable" (Tapia et al., 2013).

La conducta proecológica. Este comportamiento comprende acciones encaminadas al cuidado de recursos naturales, las cuales incluyen el reciclaje de productos, el control de desechos sólidos, el cuidado del agua, ahorro de energía, y la conservación de ecosistemas. Comprende además la lectura de temas proambientales, la persuasión pro-ecológica, el cabildeo pro-ambiental, así como el diseño y la construcción pro-ecológica (Corral et al., 2010). La investigación de este tipo de comportamiento constituye la aproximación clásica a la conducta proambiental, especialmente durante los primeros años de la psicología de la conservación. Se ha producido una buena variedad de instrumentos para medir la conducta pro-ecológica, incluyendo auto-reportes y observaciones de actos amigables con el entorno, como es el caso de la escala de Conducta Ambiental General, desarrollada por Kaiser (1998).

Conducta frugal. La frugalidad es una característica importante de un estilo de vida sustentable; ésta se caracteriza por niveles racionales de consumo, evitando el desperdicio y disminuyendo el impacto de la conducta humana en la disponibilidad y renovabilidad de los recursos naturales (De Young, 1996). Las conductas frugales antagonizan al consumismo de las sociedades modernas, el cual es una de las causas esenciales de la crisis ambiental y social (Jackson, 2008). Las acciones frugales involucran la reducción del consumo diario de productos, las decisiones acerca del tipo de productos a adquirir y consumir, las actividades en las que se involucran las personas y la manera en la que se dispone de los desechos (basura) (Tapia et al., 2013).

Conducta altruista. Desde los inicios de la investigación psico-ambiental, numerosos autores concibieron la conducta proambiental como actos altruistas; es decir, las acciones proambientales se ven como comportamientos altruistas ya que repercuten en la integridad y el bienestar de otras personas (Schultz, 2001). De hecho, se define el altruismo como una motivación dirigida a maximizar el beneficio de otros, aún si el que practica los actos altruistas gana algo o nada para sí mismo (Batson, 1991). Los comportamientos altruistas incluyen una gran gama de actos que van desde la donación de dinero a personas en necesidad, $\mathrm{o}$ a instituciones de caridad, hasta la donación de órganos y sangre y las labores de voluntariado en las que las personas regalan su tiempo para atender las necesidades de otros (Corral et al., 2010).

Conducta equitativa. La idea del desarrollo sustentable implícitamente considera la necesidad de la equidad intra e intergeneracional. Al compartir la satisfacción de necesidades entre las generaciones del presente y del futuro, el desarrollo sustentable busca un balance entre los beneficios ganados ahora por las personas y los que pueden obtenerse en los tiempos venideros (WCED, 1987). Además, ese desarrollo proclama que se debe garantizar la satisfacción de las necesidades para todos los individuos que viven en la actualidad. La equidad también implica un balance entre el bienestar humano y la integridad de los ecosistemas, lo cual hace posible para las personas el acceso a los recursos y la preservación del medio ambiente. Corral, García, Castro, Viramontes y Limones (2010) produjeron un instrumento que mide acciones de equidad social, racial, económica, de edad y género. Su estudio encontró relaciones significativas entre las acciones equitativas y las otras instancias de la conducta sustentable.

\section{Humanidad, justicia, moderación y sus respectivas fortalezas}

Al igual que las conductas sustentables, las virtudes se organizan como sistemas jerárquicos en donde una serie de fortalezas de carácter se conforman como factores de primer orden y las virtudes surgen como constructos de orden superior formados por esas fortalezas. Los factores de primer orden, a su vez, emergen o se forman a partir de rasgos o conductas observadas que se interrelacionan para constituir cada fortaleza.

La Humanidad y sus fortalezas. La virtud de la humanidad, como factor de segundo orden, está conformada por fortalezas de carácter que implican el cuidado y la amigabilidad hacia otros (Peterson \& Seligman, 2004). La amabilidad, el amor y la inteligencia social son las fortalezas 
de la humanidad y su relación con la conducta sustentable es -en teoría-bastante lógica, especialmente en lo concerniente al componente prosocial de la CS (Corral, 2012).

La amabilidad es "una motivación con base emocional que promueve conductas prosociales sin la expectativa de un beneficio recíproco" (Campos \& Algoe, 2009, p. 551), lo que vuelve a esta fortaleza un correlato del altruismo. Cairns (2005) asegura que se requiere la amabilidad para garantizar la persistencia de la fábrica social humana en esta era de degradación ecológica. A pesar de la evidente conexión entre amabilidad y conducta sustentable, queda mucho por investigar al respecto de esa relación.

El amor es concebido por Peterson y Seligman (2004) como una valoración de las relaciones cercanas con otros, manifestándose en un interés por el bienestar de la(s) persona(s) en quien(es) se deposita el amor. Para Milbrath (1996) el logro de una sociedad sustentable necesita de la confirmación del amor hacia quienes nos son más cercanos y queridos, pero también hacia las futuras generaciones, a personas en otras latitudes y a otras especies. No hay estudios que demuestren que las personas amorosas son más sustentables, lo que representa otra área de investigación a desarrollar y que aquí empezamos a emprender.

La inteligencia social implica un conjunto de habilidades que permiten desarrollar conductas sociales efectivas (por ejemplo la CS), y la conciencia de las normas grupales que afectan la conducta pro-social en diferentes situaciones; también las habilidades para seleccionar respuestas efectivas, para percibir la retroalimentación precisa de otros, y para modificar la conducta social propia, basándose en la retroalimentación recibida (Yang, 2009). Corral (2012) sugiere que es complicado que alguien sea pro-ecológico o frugal si no responde con inteligencia social a las normas que le requieren la conservación del ambiente natural y social.

Las fortalezas de la justicia. La justicia es una virtud que se apega a lo que es moralmente correcto; se puede fundamentar en la ética, la racionalidad, la ley natural, la religión o los principios de equidad. La justicia también considera el castigo a las violaciones de lo que se concibe como "correcto" (Konow, 2003). De acuerdo con Konow (op cit) una persona justa debería ser capaz de mostrar interés por el bienestar de sus semejantes, en la forma de altruismo, trabajo cooperativo, y equidad. Dentro del esquema de Peterson y Seligman (2004) las fortalezas de la justicia son la equidad, el liderazgo y el trabajo en equipo. La equidad se puede definir como "la justicia que corresponde con los derechos o las leyes naturales; más específicamente como el hecho de liberarse de los sesgos o del favoritismo" (Merriam-Webster Dictionary, edición en línea, 2011). Vimos en la sección previa que el comportamiento equitativo es una de las facetas constitutivas de la conducta sustentable. Esta fortaleza se manifiesta en actos de trato no discriminatorio a las personas; también en acciones de distribución justa de recursos entre todos. Son muy pocos los estudios que han incorporado la equidad como objeto de investigación dentro de las dimensiones psicológicas de la sustentabilidad.

El liderazgo es una fortaleza que surge en individuos que mueven a un grupo específico hacia un objetivo o misión común (Greene-Shortdridge \& Britt, 2009). Un líder comprometido con la causa de la conducta sustentable contiene el potencial de inducir el comportamiento en un número variable de personas hacia el cuidado ambiental. A este respecto, Werder (2006) asegura que el liderazgo define al activismo ambiental, y éste, a su vez, lleva a la conducta sustentable.

El trabajo en equipo, de acuerdo con Peterson y Seligman (2004), es un rasgo que se manifiesta en "trabajar bien" como miembro de un grupo o equipo. Ciertos reportes de investigación muestran la importancia del esfuerzo compartido y la cooperación en la conservación ambiental (e.g. Hargreaves, 2010). Algunos casos de mayor impacto de la conducta sustentable, como la conservación de ecosistemas o la implementación de procesos productivos no contaminantes en las empresas necesitan de manera especial el trabajo en equipo.

La moderación y sus fortalezas. La virtud de la moderación se forma a partir de fortalezas que protegen contra excesos (Peterson \& Seigman, 2004). Un componente evidente de la degradación ambiental y de los problemas sociales es la comisión de excesos: el consumismo, la sobrepoblación, y las externalidades que se manifiestan en el despilfarro de recursos y la acumulación de bienes materiales, pero también en el resentimiento, la impulsividad, y la búsqueda del placer inmediato, entre muchos otros. Ante esos excesos, los psicólogos positivos plantean el despliegue de fortalezas para minimizarlos: el perdón, la modestia, la prudencia y la auto-regulación (Corral et al., 2010). 
El perdón es una fortaleza que posibilita disminuir la indignación, la rabia o el resentimiento producidos por una ofensa real o percibida, diferencias entre personas o errores cometidos por otros (o uno mismo); el perdón también posibilita terminar con las demandas de castigo o restitución al daño real o percibido que se recibió (American Psychological Association, 2008). No se detectan estudios que investiguen si las personas con mayor capacidad de perdonar sean también individuos con mayor proclividad a la conducta proambiental, a pesar de que se sabe que quienes exhiben el rasgo del perdón tienden a ser prosociales y altruistas, no sólo con quienes los han ofendido, sino también con el resto de las personas (Karremans, Van Lange \& Holland, 2005).

La modestia es una fortaleza visible en acciones de humildad, implicando dejar que los logros propios hablen por sí mismos (Peterson \& Seligman, 2004), y evitando la auto-vanagloria o la presunción. No parecen existir estudios que hayan buscado relaciones entre la modestia y la conducta sustentable, pero es tentador suponer que por lo menos pudiera existir una asociación entre esta fortaleza y la prosocialidad, dado que una persona modesta evita que otros se sientan mal por la ostentación de bienes y capacidades propias.

La prudencia hace que las personas sean cuidadosas de sus decisiones y de no decir o hacer cosas de las que puedan después arrepentirse (Peterson \& Seligman, 2004). El buen juicio, la precaución y la anticipación o previsión caracterizan a un individuo prudente (The Free Dictionary, 2011). De Young (1991) plantea que la conducta prudente es un rasgo adaptativo de los organismos que viven en un mundo incierto, en el que no existe la seguridad de acceso ilimitado a los recursos para sobrevivir y Corral (2010) sugiere que la prudencia es un rasgo asociado a la frugalidad, una de las facetas constitutivas de la conducta sustentable. Las personas proambientales son previsoras, ya que anticipan las consecuencias de sus actos y poseen una orientación hacia el futuro (Joreiman, Van Lange, \& Van Vugt, 2004).

La auto-regulación es el auto-control que se dirige hacia un objetivo valorado, especialmente aquel objetivo que trasciende la situación inmediata (Maddux, 2009). Las personas, en grado variante, son capaces de controlar su conducta cuando buscan alcanzar metas importantes. La auto-regulación es también una forma de motivación autónoma, la cual lleva al despliegue de conductas y emociones saludables y adaptativas (Ryan \& Deci (2000). La auto-regulación se ha relacionado también con la ejecución de conductas proambientales (Pelletier, 2002; Villacorta, Koestner, \& Lekes, 2003).

\section{Objetivo del estudio}

El propósito del presente estudio fue demostrar que la conducta sustentable posee bases virtuosas, correlacionando las respuestas que dieron un grupo de individuos a un instrumento que medía acciones a favor de la sustentabilidad y tres tipos de virtudes (humanidad, justicia y moderación) incluidas en el instrumento VIA de fortalezas y virtudes (Peterson \& Seligman, 2004).

\section{Método}

\section{Participantes}

Participaron en el estudio cuatrocientas personas (150 hombres, 250 mujeres) de la ciudad de Hermosillo, México. La selección de la muestra se efectuó al azar a partir de zonas representativas del espectro socioeconómico de la ciudad, de acuerdo con los parámetros de la oficina censal mexicana INEGI (2010). La mitad de la muestra correspondió a la clase media, un cuarenta por ciento a la clase baja y el resto al estrato alto. La edad promedio de los participantes fue de $29.4(\mathrm{DE}=11)$ años, con una media de ingreso de $\$ 1,100$ (SD 899.00) dólares norteamericanos y una escolaridad promedio de $13.5(\mathrm{DE}=3)$ años de estudio.

\section{Instrumentos}

Se utilizaron los reactivos correspondientes a las virtudes de Humanidad, Justicia y Moderación del instrumento Values in Action (VIA) Inventory of Strengths (VIA-IS), versión en español. El instrumento incluye reactivos con respuestas en una escala Likert de 5 opciones ( 1 =nada parecido a mí...5=muy parecido a mí) para medir el grado de correspondencia entre el contenido de las fortalezas de carácter que comprende la clasificación VIA (Peterson \& Park, 2009; Peterson \& Seligman, 2004). Dos ejemplos de estos reactivos son "Nunca estoy demasiado ocupado cuando se trata de ayudar a un amigo", que mide amabilidad, y "Pienso que lo pasado, pasado está", que mide perdón. En la sección introductoria de este escrito se llevó a cabo una descripción de las fortalezas medidas y sus correspondientes virtudes. 
La conducta sustentable se midió a través de cuatro subescalas. La primera registra acciones altruistas a través del autoreporte de 10 conductas que refieren actos de ayuda a otros, como visitar enfermos, dar dinero a los pobres, colaborar económicamente con la Cruz Roja, etcétera. Corral et al. (2010) señalan adecuadas propiedades psicométricas para la sub-escala. Adicionalmente, se empleó el instrumento de Conducta Ecológica General de Kaiser (1998), el cual incluye el reporte de acciones como reciclar, cuidar el agua, ahorrar energía, etcétera. Estos dos instrumentos se miden con un formato de 4 opciones de respuesta $(0=$ nunca... $3=$ siempre me involucro en esta acción). Se midió también el reporte de acciones frugales, considerando aspectos como la compra de lo estrictamente necesario, la reutilización de objetos, etcétera. Corral y Pinheiro (2004) diseñaron el instrumento empleado para esta medición, el cual produjo indicadores de validez y confiabilidad. La conducta equitativa se registró con siete reactivos que indican acciones como proveer las mismas oportunidades educativas para niñas y niños, y tratar a los pobres igual que a los ricos, entre otros. Corral et al. (2010) reportan evidencias de confiabilidad, así como de validez convergente y divergente para este instrumento. Las dos últimas escalas se responden con un formato de 5 opciones de respuesta $(0=$ completamente de acuerdo $4=$ completamente en desacuerdo).

\section{Procedimiento}

Las entrevistas y llenado de instrumentos se llevaron a cabo en las viviendas de los participantes. Se obtuvo su consentimiento informado para responder a las preguntas de los instrumentos utilizados. La duración de las entrevistas fue de alrededor de 20 minutos. Todos los individuos seleccionados aceptaron participar.

\section{Análisis de datos}

Tras procesar las estadísticas univariadas para los reactivos de las sub-escalas utilizadas se obtuvieron indicadores de consistencia interna (alfas de Cronbach) para las mismas. Se computó una matriz de correlaciones entre fortalezas y conductas sustentables, y finalmente se especificó y probó un modelo estructural en el que se formaron factores de primer orden representando a las fortalezas y a las conductas sustentables, por separado. Esos factores sirvieron para formar un factor de segundo orden (FSO) a partir de las fortalezas - que fue denominado "virtudes"- y otro FSO, al que se le llamó "conducta sustentable", a partir de las interrelaciones entre los factores de conducta proecológica, frugal, altruista y equitativa. Se obtuvo dentro del modelo el valor de la covarianza entre ambos factores de segundo orden.

\section{Resultados}

La Tabla 1 presenta las medias y consistencia interna de las sub-escalas de conducta sustentable. En todos los casos, los valores del alfa de Cronbach fueron iguales o mayores a .74. Los participantes reportaron involucrarse más en conductas equitativas (media $=3.3$, de un rango de 0 a 4 ), a las que les siguieron los comportamientos frugales (media $=2.66$, rango $0 \mathrm{a} 4$ ), altruistas (media $=1.82$, rango del 0 al 3) $\mathrm{y}$, finalmente, los proecológicos (media $=1.78$, rango del 0 al 3 ).

Con respecto a las virtudes, las tres sub-escalas con las cuales se midieron alcanzaron valores de alfa mayores a .85. Considerando un rango posible de respuestas que iba del 0 al 5, el conjunto de fortalezas de la Justicia produjo los valores más altos (media $=2.95)$, seguido por el de la Humanidad (media $=2.93)$ y el de la Moderación $($ media $=2.75)$.

Para el caso de los datos del inventario VIA, se agruparon las respuestas en índices que representaban cada una de las 10 fortalezas medidas. Esto implicó promediar los resultados de todos los reactivos que medían la misma fortaleza; por ejemplo, el índice de "amabilidad" se formó a partir de la media de respuestas para los diez reactivos que indicaban esa fortaleza en particular. En el caso de las conductas sustentables, se computaron tres parcelas por constructo, de manera que esas tres parcelas sirvieran como los indicadores para cada uno de los cuatro factores de primer orden (conductas proecológica, frugal, altruista y equitativa).

Todas las fortalezas se encontraron significativamente relacionadas con los cuatro tipos de conductas sustentables, aunque el valor de las correlaciones varió, desde el más bajo de .20 (entre el amor y la conducta proecológica) hasta el superior, de .48 (entre la equidad y el comportamiento equitativo). La conducta sustentable más altamente relacionada con las fortalezas aquí estudiadas fue la equitativa: ocho de las diez correlaciones obtenidas fueron superiores a $r=.35$; la conducta altruista le siguió con cuatro correlaciones de esa magnitud, mientras que los comportamientos proecológico y frugal sólo produjeron una $r$ igual o mayor a .35 . 
Tabla 1

Estadísticas univariadas y consistencia interna de las sub-escalas que miden las cuatro instancias de la conducta sustentable

\begin{tabular}{|c|c|c|c|c|c|}
\hline CONDUCTA/Reactivos & Min & Máx & Media & DE & Alfa \\
\hline COND. PRO-ECOLÓGICA & & & 1.78 & 1.00 & 0.82 \\
\hline Carga completa en lavadora & 0 & 3 & 2.20 & 0.97 & \\
\hline Conduce a baja velocidad & 0 & 3 & 1.44 & 1.12 & \\
\hline Recicla papel usado & 0 & 3 & 1.62 & 1.13 & \\
\hline Separa botellas para reciclar & 0 & 3 & 1.40 & 1.13 & \\
\hline Llama atención por actos antiecológicos & 0 & 3 & 1.78 & 1.01 & \\
\hline Compra empaques reutilizables & 0 & 3 & 1.67 & 0.96 & \\
\hline Compra frutas de temporada & 0 & 3 & 2.21 & 0.86 & \\
\hline Lee temas ambientales & 0 & 3 & 1.63 & 0.95 & \\
\hline Conversa temas ambientales & 0 & 3 & 1.70 & 0.97 & \\
\hline Cuida energía eléctrica & 0 & 3 & 2.46 & 0.85 & \\
\hline Anima amigos a reciclar & 0 & 3 & 1.56 & 1.03 & \\
\hline Ahorra gasolina, caminando & 0 & 3 & 1.74 & 1.02 & \\
\hline COND. FRUGAL & & & 2.66 & 1.19 & 0.74 \\
\hline Conserva auto antiguo & 0 & 4 & 2.94 & 1.33 & \\
\hline Utiliza ropa temporada pasada & 0 & 4 & 3.15 & 1.08 & \\
\hline No compra joyas & 0 & 4 & 3.19 & 1.19 & \\
\hline No compra muchos zapatos & 0 & 4 & 1.89 & 1.44 & \\
\hline Evita desperdicio de comida & 0 & 4 & 2.05 & 1.34 & \\
\hline Evita comprar demasiada ropa & 0 & 4 & 1.90 & 1.43 & \\
\hline Come casi siempre en casa & 0 & 4 & 2.88 & 1.24 & \\
\hline Camina en lugar de usar coche & 0 & 4 & 2.93 & 1.28 & \\
\hline Reusa cuadernos y papel & 0 & 4 & 2.87 & 1.25 & \\
\hline Vive sin lujos, aun pudiendo & 0 & 4 & 2.81 & 1.08 & \\
\hline COND. ALTRUISTA & & & 1.82 & 0.93 & 0.78 \\
\hline Regala ropa en buen estado & 0 & 3 & 2.12 & 0.89 & \\
\hline Atiende personas que se lastiman & 0 & 3 & 2.31 & 0.81 & \\
\hline Contribuye con Cruz Roja & 0 & 3 & 2.08 & 0.88 & \\
\hline Visita enfermos en hospitales & 0 & 3 & 1.18 & 1.03 & \\
\hline Ayuda a personas mayores & 0 & 3 & 1.88 & 0.92 & \\
\hline Guía a personas buscando dirección & 0 & 3 & 2.23 & 0.80 & \\
\hline Regala moneda a indigentes & 0 & 3 & 2.00 & 0.93 & \\
\hline Participa en eventos caritativos & 0 & 3 & 1.23 & 1.13 & \\
\hline Dona sangre & 0 & 3 & 0.93 & 1.12 & \\
\hline Colabora con compañeros & 0 & 2 & 2.27 & 0.81 & \\
\hline COND. EQUITATIVA & & & 3.30 & 1.03 & 0.77 \\
\hline Pareja tiene mismos derechos & 0 & 4 & 3.31 & 1.06 & \\
\hline Trata compañeros como iguales & 0 & 4 & 3.37 & 0.98 & \\
\hline Niños toman decisiones & 0 & 4 & 2.78 & 1.21 & \\
\hline Hombres mismas obligaciones & 0 & 4 & 3.29 & 1.08 & \\
\hline Trata indígenas como iguales & 0 & 4 & 3.36 & 0.95 & \\
\hline Trata a pobres igual que a ricos & 0 & 4 & 3.44 & 0.87 & \\
\hline Niñas, oportunidades de estudiar & 0 & 4 & 3.60 & 0.91 & \\
\hline
\end{tabular}


Tabla 2

Estadísticas univariadas y consistencia interna de las sub-escalas que miden las cuatro instancias de la conducta sustentable

\begin{tabular}{lccccc}
\hline VIRTUDES/Fortalezas & Min & Máx & Media & DE & Alfa \\
\hline HUMANIDAD & & & 2.93 & 0.59 & 0.87 \\
\hline Amor & 0.90 & 4.00 & 2.96 & 0.58 & 0.78 \\
\hline Amabilidad & 1.10 & 4.00 & 3.03 & 0.58 & 0.79 \\
\hline Inteligencia social & 0.40 & 4.00 & 2.82 & 0.62 & 0.78 \\
\hline JUSTICIA & & & 2.95 & 0.61 & 0.92 \\
\hline Trabajo en equipo & 0.90 & 4.00 & 2.96 & 0.61 & 0.83 \\
\hline Equidad & 0.80 & 4.00 & 2.99 & 0.61 & 0.81 \\
\hline Liderazgo & 1.10 & 4.00 & 2.91 & 0.59 & 0.80 \\
\hline MODERACIÓN & & & 2.75 & 0.61 & 0.86 \\
\hline Perdón & 0.25 & 4.00 & 2.80 & 0.65 & 0.78 \\
\hline Modestia & 0.80 & 4.00 & 2.67 & 0.58 & 0.75 \\
\hline Prudencia & 0.78 & 4.00 & 2.75 & 0.62 & 0.80 \\
\hline Auto-regulación & 0.60 & 4.00 & 2.79 & 0.59 & 0.75 \\
\hline
\end{tabular}

Por su parte, la equidad, el liderazgo, el perdón, la prudencia y la autorregulación fueron las fortalezas que alcanzaron dos correlaciones moderadamente altas $(r=>.35)$ con comportamientos sustentables. En este marco, predominan las conexiones sobresalientes entre las fortalezas de la moderación y las conductas sustentables (ver Tabla 3).
En la figura 1 se encuentran los resultados de un modelo estructural en el que se muestra que los factores de primer orden (fortalezas, conductas sustentables) emergen de manera coherente a partir de las altas y significativas interrelaciones entre sus indicadores manifiestos. Las cargas factoriales indican validez convergente de constructo para esos factores. Puede observarse también que los factores de segundo orden

\begin{tabular}{|c|c|c|c|c|}
\hline & $\begin{array}{l}\text { Conducta } \\
\text { altruista }\end{array}$ & $\begin{array}{l}\text { Conducta } \\
\text { proecológica }\end{array}$ & $\begin{array}{c}\text { Conducta } \\
\text { frugal }\end{array}$ & $\begin{array}{l}\text { Conducta } \\
\text { equitativa }\end{array}$ \\
\hline Amor & .32 & .20 & .25 & .37 \\
\hline Amabilidad & .30 & .23 & .29 & .44 \\
\hline Inteligencia social & .32 & .31 & .24 & .38 \\
\hline Trabajo en equipo & .26 & .24 & .33 & .44 \\
\hline Equidad & .30 & .28 & .36 & .48 \\
\hline Liderazgo & .36 & .30 & .30 & .42 \\
\hline Perdón & .35 & .26 & .31 & .38 \\
\hline Prudencia & .38 & .31 & .29 & .37 \\
\hline Modestia & .34 & .27 & .26 & .28 \\
\hline Autorregulación & .38 & .39 & .31 & .27 \\
\hline
\end{tabular}




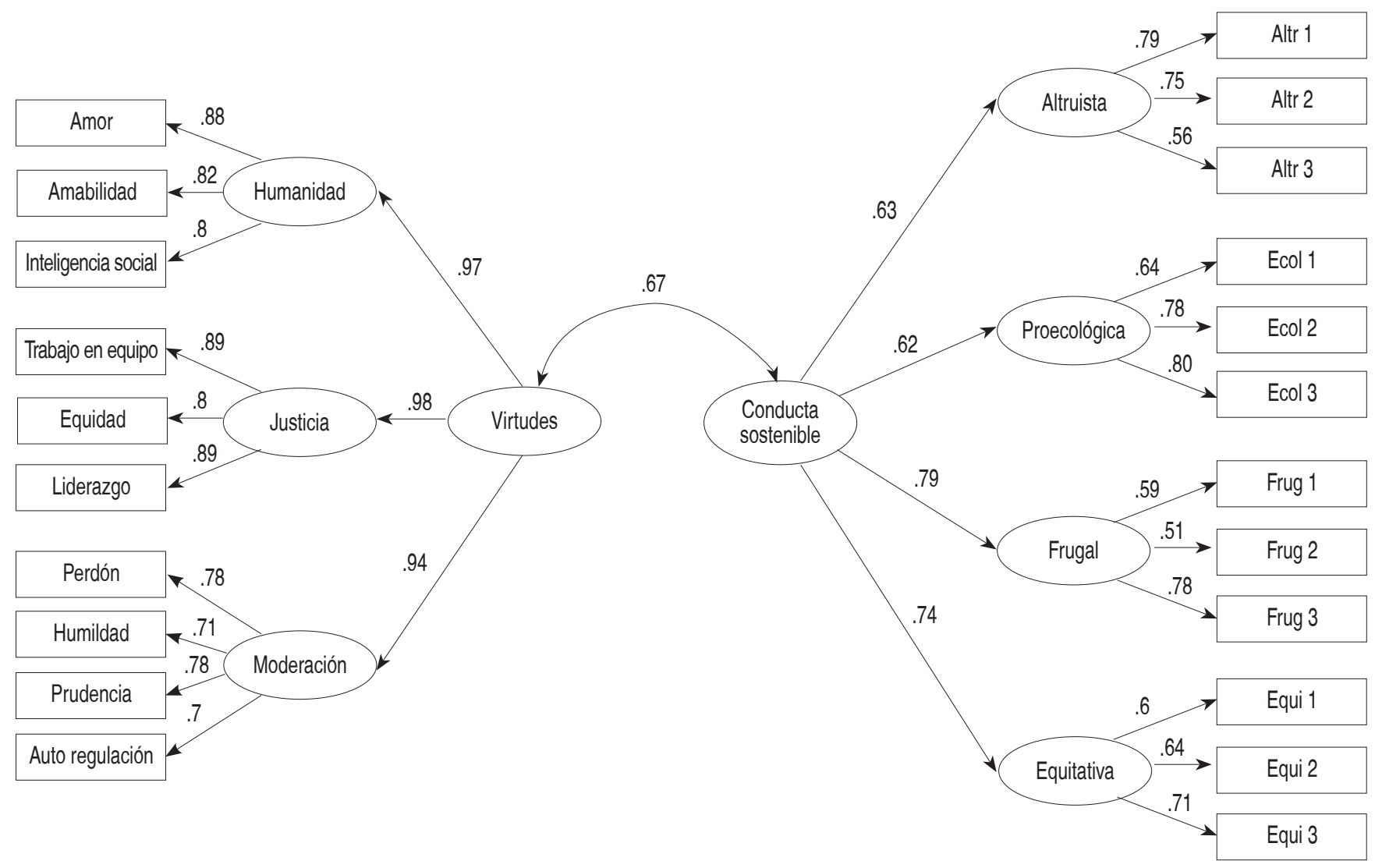

Figura 1. Modelo estructural de relaciones entre tres virtudes universales y cuatro instancias de conducta sustentable. Todas las cargas factoriales y el coeficiente de covarianza son significativos $(p<.05)$. Bondad de ajuste: $X^{2}=641.82$ (201 gl), $p<.001 ; B B N F I=.91 ; C F l=.92 ;$ RMSEA=.06.

"Virtudes" y "Conducta Sustentable" se forman a partir de los constructos de primer orden correspondientes, los cuales cargan alta y significativamente en los FSOs. La covarianza entre las virtudes y la conducta sustentable es sobresaliente y significativa $(p<.05)$. Los indicadores de bondad de ajuste del modelo se señalan al pie de la figura. Aunque la $X^{2}$ resultó ser significativa (dado el número de casos analizados), los indicadores prácticos (NNFI, CFI) así como el RMSEA indican que el modelo se ajusta adecuadamente a los datos.

\section{Discusión}

Como se esperaba, el estudio aquí reportado encontró una significativa correlación entre las virtudes de Humanidad, Justicia y Moderación y las conductas sustentables que se manifiestan en actos pro-ecológicos, frugales, altruistas y equitativos. Esta correlación no sólo se dio entre los factores de orden superior (virtudes, conductas sustentables) sino también entre todas las fortalezas particulares que integran a cada una de las virtudes aquí estudiadas, y todas las instancias de la conducta sustentable. Aunque esas correlaciones particulares variaron desde las más bajas $(r=.20)$ hasta las más notorias $(r=.48)$, todas fueron significativas. Esto refuerza la idea expuesta recientemente por Corral (2012) en el sentido de considerar a la CS como un comportamiento generalizadamente virtuoso.

En algunos casos estas correlaciones específicas respaldan hipótesis bastante lógicas, como por ejemplo, la que plantea que la conducta equitativa debe relacionarse ampliamente con la fortaleza de carácter de la equidad, u otra que especifica que las fortalezas de la autorregulación y la prudencia debieran asociarse a las conductas proecológicas, pero también a las frugales, ya que ambas se basan en buena medida en tendencias a la moderación en el uso y consumo de productos (Corral et al., 2010). Como se demostró, dichas hipótesis fueron respaldadas por los datos. Adicionalmente, otras hipótesis no comúnmente expuestas en la literatura psico-ambiental también fueron probadas y apoyadas por los resultados. 
Entre estas últimas se encuentran, por ejemplo, las hipótesis que involucran relaciones significativas de las fortalezas del amor, la amabilidad y la inteligencia social con las cuatro manifestaciones de la conducta sustentable. Si los resultados que encontramos se replican sistemáticamente en futuros estudios, se demostraría que se requiere de una buena dosis de Humanidad - en tanto virtud que nos decanta hacia el cuidado y la gentileza dirigidas a otros para ser sustentables. Esto tiene mucho sentido ya que el interés por el bienestar de los demás, como se manifiesta en los rasgos del amor, la amabilidad, y las habilidades para ser efectivos en situaciones sociales, puede fungir como acicate para el cuidado del ambiente socio-físico (Milfont \& Sibley, 2011; Schultz, 2001, Yang, 2009).

Sin embargo, resulta interesante el hecho de que las fortalezas que constituyen la Justicia y la Moderación resultaran más notoriamente relacionadas con las conductas sustentables que aquellas que están incluidas en la Humanidad. Aunque la Justicia concentró sus correlaciones más altas con la conducta equitativa -algo que era esperable- la Moderación distribuyó sus correlaciones mayores a lo largo de tres de las cuatro conductas sustentables. Destacaron aquí las conexiones entre la fortaleza del perdón y las conductas altruistas y equitativas, las cuales - hasta donde es posible detectar - no habían sido previamente investigadas. Este resultado tiene también sentido, de acuerdo con lo que la literatura anticipaba: las personas que poseen la fortaleza del perdón tienden a ser prosociales (Karremans et al., 2005) y esto parece llevarlos a practicar actos altruistas y equitativos, como parecen indicar los datos de nuestro estudio.

Mientras que el comportamiento equitativo resultó ser el más virtuoso dentro de las cuatro instancias de la $\mathrm{CS}$, las conductas frugales y las pro-ecológicas produjeron relaciones no tan notorias con las tres virtudes analizadas. Esto podría tener dos fuentes de explicación: a) una de ellas sería que la Humanidad y la Justicia tienen una connotación más pro-social que pro-ecológica ya que sus objetos de interés son fundamentalmente las personas. Sin embargo, la Moderación debiera tener implicaciones evidentes en la mitigación del consumismo (como tendencia opuesta a la frugalidad) y en la depredación de recursos naturales (como antagonista de los actos pro-ecológicos). Es posible, en este caso, que los individuos estén incorporando en su visión del mundo la idea de que la prudencia y la autorregulación se asocian más al control de impulsos en situaciones (pro) sociales -algo que resulta evidente en el caso del perdón y la modestia- que al autocontrol en el uso de recursos naturales. Sin embargo, debe resaltarse como lo enunciamos previamente que la auto-regulación sí resultó notoriamente asociada a la conducta pro-ecológica. b) Otra posible explicación sería que las personas no detectan tanta virtud en los actos de cuidado del ambiente natural, como en las acciones de cuidado de sus semejantes. Si este último es el caso, dicha situación representa un área de oportunidad para la educación ambiental.

Tomadas en conjunto, las tres virtudes se asocian significativamente al conjunto de comportamientos sustentables, de acuerdo con lo que arrojan los resultados del modelo estructural. Estos resultados sugieren que el desarrollo de virtudes en la formación cívica-especialmente entre jóvenes- podría ofrecer una vía de instigación de la conducta sustentable. El tamaño de la relación entre estas tres virtudes y la CS (covarianza $=.67$ ) habla del peso que podría tener el despliegue de fortalezas y virtudes en la conducta pro-social y proambiental de los ciudadanos.

Por supuesto, debe reconocerse que al haberse recogido los datos mediante el empleo de auto-reportes una parte de las respuestas de los participantes podría deberse al sesgo de la deseabilidad social (las personas podrían tender a brindar información que los hace parecer más virtuosos, pro-sociales y proambientales que lo que realmente son). Los auto-reportes recogen mejor información relacionada con actitudes que con conductas. Esta situación se presenta como una posible limitación de nuestra investigación. Otra limitación es que sólo se investigaron tres de las seis virtudes universales incluidas en la clasificación de Peterson y Seligman (2004).

A pesar de esto, el estudio aquí descrito ofrece un área relativamente original y potencialmente provechosa en la investigación de los determinantes de la conducta sustentable: un campo que pretende indagar la naturaleza virtuosa de esa conducta y las maneras en las que el desarrollo de las virtudes universales pudiera influir en el surgimiento y mantenimiento de acciones favorables al entorno social y físico.

\section{Referencias}

American Psychological Association (2008). Forgiveness: a sampling of research results. Washington, D.C.: A.P.A.

Batson, C.D. (1991). The Altruism Question: Toward a Social Psychological Answer (p. 80). New Hillsdale, NY: Erlbaum. 
Brown, K.W., \& Kasser, T. (2005). Are psychological and ecological well-being compatible? The role of values, mindfulness, and lifestyle. Social Indicators Research, 74, 349-368. doi: 10.1007/s11205-004-8207-8

Cairns, J. (2005). Can compassion and kindness survive a violent cutback in human population size? Recuperado de: http://www.johncairns.net/ Commentaries/8novcomm.pdf.

Campos, B., \& Algoe, S. (2009). Kindness. En S.J. López (Ed.), The Encyclopedia of Positive Psychology (pp. 551-557). Chichester, UK: Wiley-Blackwell.

Corral, V. (2010). Psicología de la Sustentabilidad. Un análisis de lo que nos hace proecológicos y prosociales. México: Editorial Trillas.

Corral, V. (2012). The positive Psychology of Sustainability. Environment, Development and Sustainability, 14, 651-666. doi:10.1007/s10668-012-9346-8

Corral, V. Frías, M., \& García, C. (2010). Introduction to the psychological dimensions of sustainability. En V. Corral, C. García, y M Frías (Eds). Psychological Approaches to Sustainability (pp. 3-18). New York: Nova Science Publishers.

Corral, V., García, C., Castro, L., Viramontes, I., \& Limones, R. (2010). Equity and sustainable lifestyles. En V. Corral, M. Frías, \& C. García (Eds.), Psychological Approaches to Sustainability (pp. 185-204). New York: Nova Science Publishers.

Corral, V., y Pinheiro, V. (2004). Aproximaciones al estudio de la conducta sustentable. Medio Ambiente y Comportamiento Humano, 5, 1-26.

De Groot, J., \& Steg, L. (2007). Value orientations and environmental beliefs in five countries: Validity of an instrument to measure egoistic, altruistic, and biospheric value orientations. Journal of Crosscultural Psychology, 38, 318-332. doi: $10.1177 / 0022022107300278$

De Young, R. (1991). Some psychological aspects of living lightly: Desired lifestyle patterns and conservation behavior. Journal of Environmental Systems, 20, 215-227. doi: 10.2190/030Q-Q4KE-7YFB-4Q0F

De Young, R. (1996). Some psychological aspects of a reduced consumption lifestyle: The role of intrinsic satisfaction and competence motivation, Environment \& Behavior, 28, 358-409. doi: 10.1177/0013916596283005
Dobson, A. (2007). Environmental citizenship: towards sustainable development. Sustainable Development, 15, 276-285. doi: 10.1002/sd.344

Dunlap, R.E., Grieneeks, J., \& Rokeach, M. (1983). Human values and pro-environmental behavior. En W.D. Conn (Ed.), Energy and material resources: Attitudes, values and public policy (pp. 145-168). Boulder, CO: Westview Press.

Fowers, B. J. (2005). Virtue and psychology: Pursuing excellence in ordinary practices. Washington, DC: APA Press.

Greene-Shortdridge, T., \& Britt, T. (2009). Leadership. Fowers, B. (2009). Open-mindeness. En S.J. López (Ed.), The Encyclopedia of Positive Psychology (pp. 567-574). Chichester, UK: Wiley-Blackwell.

Hargreaves, T. (2010). Putting Foucault to work on the environment: exploring pro-environmental behavior change as a form of discipline. CSERGE Working Paper EDM 10-11. Recuperado de: http://eastanglia. academia.edu/TomHargreaves/Papers/243719Putting_Foucault_to_work_on_the_environment_exploring proenvironmental_behaviour_change_as_a_form_ of_discipline.

INEGI. (2010). Estados Unidos Mexicanos. Principales resultados por localidad. XII Censo General de Población y Vivienda. Mexico, DF: Autor.

Jackson, T. (2008). The challenge of sustainable lifestyles. En L. Starke (Ed.), State of the World (pp. 45-60). New York: W.W. Norton and Company.

Joreiman, J. A., Van Lange, P. A. \& Van Vugt, M. (2004). Who cares about the environmental impact of cars? Those with an eye toward the future. Environment \& Behavior, 36, 187-206. doi: 10.1177/0013916503251476

Kaiser, F. (1998). A general measure of ecological behavior. Journal of Applied Social Psychology, 28, 395-442. doi: 10.1111/j.1559-1816.1998.tb01712.x

Karremans, J., Van Lange, P. \& Holland, R. (2005). Forgiveness and its association with prosocial thinking, feeling, and doing beyond the relationship with the offender. Personality and Social Psychology Bulletin, 31, 1315-1326. doi: 10.1177/0146167205274892

Konow, J. (2003). Which is the fairest one of all? A positive analysis of Justice Theories. Journal of Economic Literature, 61, 1188-1239. doi: $10.1257 / 002205103771800013$ 
Maddux, J. (2009). Self-regulation. En S.J. López (Ed.), The Encyclopedia of Positive Psychology (pp. 889-893). Chichester, UK: Wiley-Blackwell.

Merriam-Webster Dictionary (2011). Equity. Recuperado de: http://www.merriam-webster.com/dictionary/ equity.

Milbrath, L. (1996). Envisioning a sustainable society. En R. Slaughter (Ed.), New thinking for a new millennium (pp. 185-196). New York: Routledge.

Milfont, T., \& Sibley, C. (2011). Exploring the concept of environmental generativity. International Journal of Hispanic Psychology, 4, 21-30.

Nilsson, A., von Borgstede, C., \& Biel, A. (2004). Willigness to accept climate change policy measures: The effect of values and norms. Journal of Environmental Psychology, 24, 267-277. doi: 10.1016/j.jenvp.2004.06.002

Pelletier, L. G. (2002). A motivational analysis of selfdetermination for pro-environmental behaviors. En E. L. Deci \& R.M. Ryan (Eds.), Handbook of self-determination research (pp. 205-232). Rochester, NY: University of Rochester Press.

Peterson, C., \& Park, N. (2009). Classifying and measuring strengths of character. In S. J. Lopez \& C. R. Snyder (Eds.), Oxford handbook of positive psychology, 2nd edition (pp. 25-33). New York: Oxford University Press.

Peterson, C., \& Seligman, M. (2004). Character strengths and virtues: a handbook and classification. Washington, DC: American Psychological Association.

Roseland, M. (2012). Toward sustainable communities. Gabriola Island, Canada: New Societies Publishers.

Ryan R., \& Deci, E. (2000). Self-determination theory and the facilitation of intrinsic motivation, social development, and well-being. American Psychologist, 55, 68-78. doi: 10.1037/0003-066X.55.1.68
Schultz, P.W. (2001). The structure of environmental concern. Concern for self, other people, and the biosphere. Journal of Environmental Psychology, 21, 327-339. doi: 10.1006/jevp.2001.0227

Steg, L., \& de Groot, J. (2012). Environmental values. En S. Clayton (Ed.), The Oxford Handbook of Environmental and Conservation Psychology. (pp. 81-92). New York: Oxford University Press.

Steg, L., Vlek, C., \& Slotegraaf, G. (2001). Instrumentalreasoned and symbolic-affective motives for using a motor car. Transportation Research-F: Psychology and Behaviour, 4, 151-169. doi: 10.1016/S13698478(01)00020-1

Tapia, C., Corral, V., Fraijo, B., \& Durón, F. (2013). Assessing sustainable behavior and its correlates: a measure of pro-ecological, frugal, altruistic and equitable actions. Sustainability, 5, 711-723. doi: 10.3390/su5020711

The Free Dictionary (2011). Prudence. Recuperado de: http://www.thefreedictionary.com/prudence.

Villacorta, M., Koestner, R., \& Lekes, N. (2003). Further validation of the Motivation toward the Environment Scale. Environment \& Behavior, 35, 486-505. doi: 10.1177/0013916503035004003

WCED World Commission on Environment \& Development (1987). Our Common Future; Oxford, UK: Oxford University Press.

Werder, O. (2006). Influences on the recycling behavior of young adults: avenues for social marketing campaigns. En P. Depoe (Ed.), The Environmental Communication Yearbook, Vol. 3 (pp. 77-96). Mahwah, New Jersey: Erlbaum.

Yang, Y. (2009). Social skills. En S.J. López (Ed.), The Encyclopedia of Positive Psychology (pp. 912-913). Chichester, UK: Wiley-Blackwell. 